\title{
Development of a Multiscale Thermal Conductivity Model for Fission Gas in $\mathrm{UO}_{2}$
}

\author{
Michael R Tonks ${ }^{\mathrm{a}, \mathrm{b}, *}$, Xiang-Yang Liuc ${ }^{\mathrm{c}}$, David Andersson ${ }^{\mathrm{c}}$, Danielle Perez ${ }^{\mathrm{b}}$, Aleksandr Chernatynskiy ${ }^{\mathrm{d}}$, \\ Giovanni Pastore ${ }^{\mathrm{b}}$, Christopher R Stanek ${ }^{\mathrm{c}}$, Richard Williamson ${ }^{\mathrm{b}}$ \\ ${ }^{a}$ Department of Mechanical and Nuclear Engineering, Pennsylvania State University, University Park, PA 16802. \\ ${ }^{b}$ Fuel Modeling and Simulation, Idaho National Laboratory, P.O. Box 1625, Idaho Falls, ID 83415. \\ ${ }^{c}$ Materials Science and Technology Division, Los Alamos National Laboratory P.O. Box 1663, Los Alamos, NM 87545 \\ ${ }^{d}$ Department of Physics, Missouri University of Science and Technology, Rolla, MO 65409
}

\begin{abstract}
Accurately predicting changes in the thermal conductivity of light water reactor $\mathrm{UO}_{2}$ fuel throughout its lifetime in reactor is an essential part of fuel performance modeling. However, typical thermal conductivity models from the literature are empirical. In this work, we begin to develop a mechanistic thermal conductivity model by focusing on the impact of gaseous fission products, which is coupled to swelling and fission gas release. The impact of additional defects and fission products will be added in future work. The model is developed using a combination of atomistic and mesoscale simulation, as well as analytical models. The impact of dispersed fission gas atoms is quantified using molecular dynamics simulations corrected to account for phonon-spin scattering. The impact of intragranular bubbles is accounted for using an analytical model that considers phonon scattering. The impact of grain boundary bubbles is determined using a simple model with five thermal resistors that are parameterized by comparing to 3D mesoscale heat conduction results. However, when used in the BISON fuel performance code to model four reactor experiments, it produces reasonable predictions without having been fit to fuel thermocouple data.
\end{abstract}

Keywords: Fuel Performance Modeling, Multiscale Modeling, Uranium Dioxide, Thermal Conductivity

\section{Introduction}

Light water reactors (LWRs) generate electricity from heat produced from fissioning atoms within $\mathrm{UO}_{2}$ fuel pellets. The thermal conductivity of the fuel defines how efficiently heat is transported from the fuel to the coolant, and therefore has a direct impact on the production of electricity. Unfortunately, the same fission events that generate heat also cause radiation damage that decreases the thermal conductivity and thus the efficiency of heat removal from the fuel. This reduction in thermal conductivity not only reduces the efficiency of the reactor, but also its safety, as excess heat generated during an accident cannot be removed from the fuel, resulting in high temperatures that can cause the fuel to melt.

\footnotetext{
${ }^{*}$ Corresponding author

Email address: mrt5296@psu.edu (Michael R Tonks)
}

Preprint submitted to Elsevier
Radiation damage within the fuel causes a range of microstructural changes, including point defect generation, dislocation loop formation, fission product generation, and bubble formation. All of these changes decrease the thermal conductivity (with the exception of the formation of metallic precipitates). These same microstructural changes cause a range of other effects, including swelling, embrittlement, and so on. Thus, all these various changes in the properties are interrelated and are due to changes in the fuel microstructure.

Due to the impact of the fuel thermal conductivity on reactor efficiency and safety, significant efforts have been spent to understand its behavior as a function of temperature and burn-up. Numerous experimental measurements of the thermal conductivity in unirradiated $\mathrm{UO}_{2}$ have led to the development of various semi-empirical models of the fresh $\mathrm{UO}_{2}$ thermal conductivity, summarized by Fink [1]. Additional data have been collected on the ther-

November 10, 2015 
mal conductivity of irradiated $\mathrm{UO}_{2}$, and a range of different models have been developed that determine the degradation of the thermal conductivity as a function of burn-up [2-4]. While these empirical models perform well within the conditions at which the data was collected, they cannot be extrapolated outside those conditions. In addition, while the burn-up is a measure of the amount of fissioning that has taken place, it cannot account for the impact of the operating conditions on the fuel microstructure, which directly impacts the thermal conductivity.

In an ongoing effort, we are replacing the empirical materials models for fuel performance with a series of interdependent microstructure based models. In this approach, a series of variables that define the current state of the microstructure evolve with time, as a function of the temperature, stress, and other state variables. The material properties of the fuel evolve as a function of these state variables. While some information required to develop such a modeling approach can be obtained from experimental data, most would be very difficult and expensive to obtain experimentally. Thus, we reduce the amount of required data using multiscale modeling and simulation. In this approach, atomistic simulations identify critical mechanisms and determine key material property values, mesoscale models developed in the MARMOT mesoscale fuel performance code [5] use the atomistic information to predict the coevolution of microstructure and properties, and the mesoscale results inform the development of mechanistic materials models defining the evolution of the state variables and how they impact the material properties. This approach is also discussed in Tonks et al. [6].

One important aspect of the in-reactor operation of the fuel is the creation of gaseous fission products. Such fission gas degrades the fuel thermal conductivity, but also causes swelling and can be released into the gap and plenum, increasing pressure and decreasing gap thermal conductivity. Models have been developed to predict the swelling and fission gas release, but these models are independent of each other and of the thermal conductivity model, even though in reality the effects are coupled. The lack of coupling does not cause problems during steady reactor operation, as the models are typically calibrated together, but would negatively impact the results outside of the conditions where the calibration was conducted. In our new microstructure-based approach to fuel performance modeling, the current state of the fission gas is described by several state variables. These variables have already been coupled to the swelling model $[7,8]$, and some initial efforts have been made to couple fission gas found in bubbles on grain boundaries to changes in the thermal conductivity [6]. However, additional efforts are needed to account for the impact of other distributions of fission gas.

The objective of the present study is to create a model that fully accounts for the impact of gaseous fission products on the fuel thermal conductivity, and directly couples to the variables defining the current state of the fission gas evolved using the fission gas behavior model from Pastore et al. [7, 8]. A combination of experimental data, atomistic simulation, and mesoscale simulation are used to elucidate the impact of the various distributions of fission gas within the fuel. Each of these contributions are combined in a thermal conductivity model that is implemented in the macroscale fuel performance code BISON [9] and compared against experimental data. However, this model only accounts for the contribution of fission gas on thermal conductivity. Future work will add the contributions of other fission products and defects to the thermal conductivity model.

We begin in Section 2 by summarizing the fission gas release model that is used to quantify the different distributions of fission gas. We then discuss the development of our fission gas thermal conductivity model in Section 3, where the impact of dispersed fission gas is discussed in Section 3.1, of intragranular gas bubbles in Section 3.2, and of grain boundary (GB) bubbles in Section 3.3. We end in Section 4 summarizing how we implemented the model in the BISON fuel performance code and evaluate the model by comparing to data and to other fuel performance codes.

\section{Fission Gas Model Summary}

Gaseous fission products are produced throughout the fuel. The fission gas eventually migrates to GBs, forming networks of intergranular bubbles. As the gas atoms migrate through the grain interiors, small intragranular bubbles form, preventing some fission gas from reaching the GBs. Thus, fission gas can be divided into three main categories: fission gas in intergranular bubbles, gas in intragranular bubbles, and gas atoms dispersed within the grains that are still mobile. 
In the models from Pastore et al. [7], Speight [10], White and Tucker [11], the gas atom behavior is considered in an idealized spherical grain. For the one-dimensional geometry, the intragranular diffusion of fission gas is defined by

$$
\frac{\partial C_{t}}{\partial t}=D_{e f f} \frac{1}{r^{2}} \frac{\partial}{\partial r}\left(r^{2} \frac{\partial C_{t}}{\partial r}\right)+\beta,
$$

where $C_{t}$ is the total intragranular gas atom concentration (including gas both in and out of bubbles), $t$ is the time, $D_{\text {eff }}$ is the effective gas diffusivity, $r$ is the radial coordinate and $\beta$ is the gas generation rate. The effective diffusivity accounts for trapping in and irradiation-induced resolution from intragranular bubbles, according to

$$
D_{e f f}=\frac{b}{b+g} D_{s},
$$

where $g$ is the rate of gas atom trapping, $b$ is the resolution rate from the bubble back into the lattice, and $D_{s}$ is the gas atom diffusivity. Equation (2) assumes that the bubbles are saturated, giving

$$
\frac{C_{s}}{C_{b}}=\frac{b}{g}
$$

where $C_{s}$ is the concentration of dispersed intragranular gas and $C_{b}$ is the concentration of gas in intragranular bubbles, with

$$
C_{t}=C_{s}+C_{b} .
$$

The trapping and resolution rates are determined from

$$
\begin{aligned}
g & =4 \pi D_{s} R_{i g} N_{i g} \\
b & =3.03 F \pi l_{f}\left(R_{i g}+Z_{0}\right)^{2},
\end{aligned}
$$

where $R_{i g}$ is the mean radius of intragranular bubbles, $N_{i g}$ is the number density of intragranular bubbles, $F$ is the fission density, $l_{f}$ is the fission fragment track length, and $Z_{0}$ is the radius of influence of the fragment track. The bubble radius and number density are calculated as

$$
R_{i g}=5 \times 10^{-10}\left(1+106 e^{\frac{1.20 \times 10^{-19}}{-k_{b} T}}\right)
$$

and

$$
N_{i g}=\frac{1.52 \times 10^{27}}{T}-3.3 \times 10^{23}\left(\mathrm{~m}^{-3}\right),
$$

with $k_{b}$ being the Boltzmann constant in $\mathrm{J} / \mathrm{K}$ and $T$ the temperature in $\mathrm{K}$ [11]. The intragranular bubble porosity is determined according to

$$
p_{i g}=N_{i g}\left(\frac{4}{3} \pi R_{i g}^{3}\right) .
$$

The model of the intergranular bubble development is described in detail in Pastore et al. [7, 8]. To summarize, the main assumptions of the model are: the effects of GB triple junctions are neglected, an initial number density of intergranular bubbles is considered, and further nucleation is disregarded. The arrival rate of gas atoms to the GB is assumed equal to the gas absorption rate at intergranular bubbles. In addition, all intergranular bubbles are assumed to be of equal size and shape, and resolution from GBs is neglected. The model calculates the coupled fuel gaseous swelling, and fission gas release by describing the basic mechanisms of intergranular bubble growth with inflow of gas atoms and vacancies, bubble coalescence, and bubble interconnection.

The GB bubble fractional coverage is calculated as

$$
f_{c}=N_{g b} A_{g b},
$$

where $N_{g b}$ is the number density of GB bubbles and $A_{g b}$ is the average projected bubble area on the GB. The bubble number density decreases with time due to coalescence as the bubble area increases in the growth process. When the fractional coverage reaches a saturation value, $f_{c, s a t}$, further bubble growth is compensated by fission gas release and the saturation condition $f_{c}=N_{g b} A_{g b}=f_{c, s a t}$ holds. The calculation of $N_{g b}$ and $A_{g b}$ is detailed in Pastore et al. [7, 8]. Also, we adopt the model extension proposed in [12] to account for transient (burst) release. Such extension introduces an additional gas release contribution as the grain boundary gas storing capacity, and consequently $f_{c}$ and $\left.f_{c, s a t}\right)$, decreases during temperature transients due to fuel micro-cracking.

Thus, the model defines the fission gas behavior in terms of three state variables: the fractional coverage of bubbles on the GB $f_{c}$ evolved according to Eq. (10), the intragranular bubble porosity $p_{i g}$ evolved according to Eq. (9), and the dispersed intragranular gas atom concentration $C_{s}$ evolved using

$$
C_{s}=\frac{b}{g+b} C_{t}
$$

derived from Eqs. (3) and (4).

\section{Thermal Conductivity Model}

All three categories of fission gas discussed in the previous section inhibit the transfer of heat within 
$\mathrm{UO}_{2}$, and thus lower the effective thermal conductivity. However, the impact of each category of fission gas on the thermal conductivity is different. Therefore, to create a comprehensive model of the impact of fission gas on thermal conductivity, we consider the impact of GB bubbles, intragranular bubbles and dispersed intragranular gas atoms individually.

The unirradiated thermal conductivity $k_{0}(T)$ in $\mathrm{UO}_{2}$ includes phonon lattice and small polaron ambipolar (applying equally to positive and negative ions) contributions [13], such that

$$
k_{0}(T)=k_{L}(T)+k_{A P}(T),
$$

where $k_{L}$ quantifies the phonon lattice contribution and $k_{A P}$ the small polaron contribution. The small polaron contribution was determined for $\mathrm{UO}_{2}$ by Ronchi et al. [13] by parameterizing a theoretical equation and fitting to experimental data to obtain

$$
k_{A P}(t)=\frac{6400}{t^{5 / 2}} \exp \left(\frac{-16.35}{t}\right),
$$

where $t=T(\mathrm{~K}) / 1000 \mathrm{~K}$. It significantly contributes to the thermal conductivity at temperatures $T>$ $1500 \mathrm{~K}$. The lattice thermal contribution can be approximated as

$$
k_{L}(T)=\left(A+B T+C T^{2}\right)^{-1}
$$

for temperatures above the Debye temperature, where the athermal term $A$ captures the impact of phonon-defect scattering, $B$ captures the impact of three phonon scattering [3], and $C$ captures the effect of four phonon scattering [14]. The statistical behavior of the experimental fit from Ronchi et al. [13] indicated that the four phonon scattering term should not be neglected and Fink [1] obtained an improved fit to a large set of data when using the four phonon scattering term. The fitted coefficients from Fink [1] are $A=7.5408 \times 10^{-2}$, $B=17.692 \times 10^{-2}$, and $C=3.6142 \times 10^{-2}$.

During reactor operation, small defects are generated within the fuel that add additional phonondefect scattering, lowering the thermal conductivity from the unirradiated $k_{0}(T)$ value. This additional phonon scattering is accounted for by adding a series of athermal terms to Eq. (14) such that

$$
k_{L}(T)=\left(A+B T+C T^{2}+\sum_{i} D_{i} c_{i}\right)^{-1},
$$

where $c_{i}$ is the atomic concentration of point defect $i$ and $D_{i}$ is a corresponding coefficient. In addition, secondary phases are created within the fuel that have a different local thermal conductivity than the $\mathrm{UO}_{2}$ matrix. Fission gas bubbles have a much lower thermal conductivity than the $\mathrm{UO}_{2}$ matrix, lowering the effective thermal conductivity of the microstructure, while metallic precipitates have a higher thermal conductivity and thus increase the effective thermal conductivity. The impact of secondary phases on the thermal conductivity are accounted for with "mixing" type models where the various phases are considered as thermal resistors in parallel or series or some combination of the two and come in the form of a multiplier of the bulk thermal conductivity $k_{0}(T)$. One commonly used mixing model is the Maxwell-Eucken equation [15].

Our thermal conductivity model accounts for distributed fission gas atoms by adding a scattering term to the unirradiated thermal conductivity equation. The intragranular gas bubbles are accounted for using a mixing model via a multiplier. Finally, the intergranular gas bubbles are accounted for by increasing the thermal resistance of the GBs as a function of the GB fractional coverage $f_{c}$. We summarize the development of each of these models, below.

\subsection{Modeling the Effect of Dispersed Fission Gas Atoms}

The gas atoms dispersed within the $\mathrm{UO}_{2}$ grains decrease the thermal conductivity because they increase phonon-defect scattering. Though the fission gas is actually composed of a range of different elements, the dominant type is xenon (Xe) because it has the largest concentration and causes the largest lattice distortion. Thus, for the rest of our efforts in this work, we consider only $\mathrm{Xe}$ as the fission gas. Xe has a low solubility in $\mathrm{UO}_{2}$, and thus tends to segregate to GBs and voids. When it is in the bulk, it is most commonly found in Schottky defects (composed of two oxygen vacancies and one uranium vacancy).

As we are focusing only on the impact of fission gas on the thermal conductivity, we neglect other defect types such that Eq. (15) becomes

$$
k_{L}(T)=\left(A+B T+C T^{2}+K_{X e} c_{X e}\right)^{-1} .
$$

The coefficient $K_{X e}$ can be obtained by an analytical equation [3]

$$
K_{X e}=\frac{\pi^{2} \bar{V} \theta_{D}}{3 \bar{v}^{2} h} \Gamma_{X e},
$$


where $\bar{V}$ is the mean atomic volume of the $\mathrm{UO}_{2}$ lattice, $\theta_{D}$ is the Debye temperature, $\bar{v}$ is the acoustic velocity, $h$ is Planck's constant, and $\Gamma_{X e}$ is the phonon diffusion cross-section of the fission gas. The parameter $\Gamma_{X e}$ depends on the local perturbation of mass, strain, and bonding potential of a single Xe atom. It can be approximated as

$$
\Gamma_{X e}=\frac{\bar{M}_{U O_{2}}-M_{X e}}{\bar{M}_{U O_{2}}}+\epsilon \frac{\bar{r}_{U O_{2}}-r_{X e}}{\bar{r}_{U O_{2}}},
$$

where $\bar{M}_{U_{O_{2}}}$ is the atomic weight of a $\mathrm{UO}_{2}$ molecule, $M_{X e}$ is the atomic weight of a Xe atom, $\bar{r}_{\mathrm{UO}_{2}}$ is the ionic radius of a $\mathrm{UO}_{2}$ molecule, and $r_{X e}$ is the ionic radius of a Xe atom. The constant $\epsilon=32(1+1.6 \gamma)^{2}$, where $\gamma$ is the Gruneisen constant.

We use the above expressions to calculate $K_{X e}$. We use the parameters $\bar{V}=l_{c}^{3} / 12$, with the lattice constant $l_{c}=0.547 \mathrm{~nm}$, and $\theta_{D}=385 \mathrm{~K}[16]$, $\bar{v}=2800 \mathrm{~m} / \mathrm{s}[16]$, and $\gamma=1.8$ [16]. In addition, $\bar{M}_{U O_{2}}=M_{U}+2 M_{O}$ and $\bar{r}_{U O_{2}}=l_{c}^{3} / 4$. The atomic weight $M_{X e}=131.293 \mathrm{amu}$ and the ionic radius $r_{X e}=190 \mathrm{pm}$. Using these values in Eq. (18) gives the value $K_{X e}=20.3 \mathrm{mK} / \mathrm{W}$.

To evaluate the accuracy of this model, we use molecular dynamics (MD) simulations to predict the effective thermal conductivity of $\mathrm{UO}_{2}$ single crystals with increasing densities of dispersed Xe atoms. The non-equilibrium MD method, often referred to as the "direct method," was used for the thermal conductivity simulations [17-19]. The simulations were conducted using the LAMMPS package $[20]$. In this method, a heat current $(J)$ is imposed on the system, and the thermal conductivity $(k)$ is calculated from the time-averaged temperature gradient $(\partial T / \partial z)$ applying Fourier's law, $k=-J / \partial T / \partial z$. An elongated supercell containing $3 \times 3 \times n_{z}$ cubic unit cells of $\mathrm{UO}_{2}$ (where $n_{z} \gg 3$ ) is constructed for the simulations and periodic boundaries are applied in all three dimensions. The heat flow is imposed along the $z$-direction of the cell. Heat is removed from a region one lattice constant wide at $z=0$ and heat is added to a region of the same width at $z=\frac{L_{z}}{2}$, where $L_{z}$ is the periodic length of the supercell in the $z$ direction. Note that there is only a weak dependence of the calculated thermal conductivity on cross sectional area [21]. The length $L_{z}$ in the heat flow direction ranges from 19 to $65 \mathrm{~nm}$, see below for details of the extrapolation scheme applied to avoid effects of finite simulation box length.
The simulation procedure starts by carrying out NPT (constant number, pressure, and temperature) simulations to determine the thermal expansions at different temperatures. The supercell dimensions were averaged over 100 ps. After determining the thermal expansion, the system is equilibrated for $50 \mathrm{ps}$ in the NVT (constant number, volume, and temperature) ensemble at the desired temperature, followed by another $50 \mathrm{ps}$ in the NVE (constant number, volume, and energy) ensemble. After that, non-equilibrium MD runs were performed for a period of $5-10$ ns. These relatively long atomistic simulation times ensure converged results. To calculate the thermal conductivity, the temperature profiles were averaged after the initial $0.5 \mathrm{~ns}$ (to neglect early transient behavior). The method of Jund and Jullien [17] was adopted to control the heat flux. The heat flux was set to $1.9-3.7 \times 10^{-4}$ $\mathrm{eV} / \mathrm{nm}^{2} \mathrm{fs}$.

In the relatively short supercells accessible in MD simulations, a significant portion of phonons propagate ballistically through the system and scatter from the hot and cold plates. This causes the thermal conductivity obtained from MD simulations to be lower than they should be, especially at low temperature. The conventional method [22] to extrapolate the value to an infinitely large system is based on a linear formula, $1 / \kappa=1 / \kappa_{\infty}+c / L_{z}$, where $c$ is a constant related to scattering by the hot and cold plates, $L_{z}$ is the length of the simulation cell and $\kappa_{\infty}$ is the thermal conductivity for a simulation cell of infinite length. However, in Gofryk et al. [23] a quadratic correction was needed in order to take non-linear effects of the $\mathrm{UO}_{2}$ lattice into account, which brings the fitting formula to a more general one, $1 / \kappa=1 / \kappa_{\infty}+c_{1} / L_{z}+c_{2} / L_{z}^{2}$, where $c_{1}$ and $c_{2}$ are both constants. At each temperature, we used five different box sizes for the extrapolation, 19, 24, 32, 49 and $65 \mathrm{~nm}$ in length.

MD simulations for pure $\mathrm{UO}_{2}$ were carried out using the potential from Busker et al. [24], at temperatures ranging from $200 \mathrm{~K}$ to $1500 \mathrm{~K}$. These MD results consistently overpredict the thermal conductivity of $\mathrm{UO}_{2}$, as has been previously observed in the literature $[25,26]$. This is primarily due to resonant scattering of phonons by spins in the paramagnetic phase of $\mathrm{UO}_{2}[23,27]$, which is not captured by the MD simulations. However, we can correct this deficiency by using the phonon-spin scattering contribution derived from experimental data [23]. In Ref. [23] this was achieved by fitting a Callaway model [28], including a term for the phonon- 


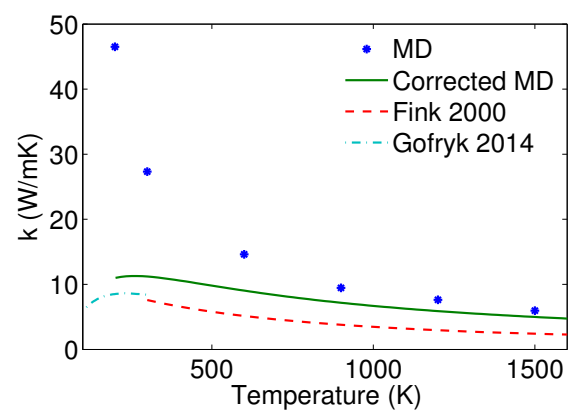

Figure 1: Comparison between the single crystal thermal conductivity calculated using MD and the values corrected using the spin scattering term from the Callaway model. The experimental fit from Fink [1] and Gofryk et al. [23] is also shown for reference.

spin scattering relaxation time [29], to experimental data collected on oriented $\mathrm{UO}_{2}$ single crystals. The phonon-spin relaxation time is then added to the total relaxation time derived by fitting the MD results using the same Callaway model without the phonon-spin scattering contribution. Since classical $\mathrm{MD}$ is valid only at temperatures comparable to the Debye temperature and above due to lack of quantum effects, MD data in that range was used for the fit. Adding the phonon-spin relaxation mechanism to the MD results provides more realistic values for the thermal conductivity, as shown in Fig. 1. The corrected values are still higher than the experimental fit for polycrystalline sintered samples from [1], but they are much closer than the uncorrected MD results. They are also closer to the single crystal data from [23]. The remaining discrepancy between simulations and experiments can most likely be attributed to uncertainties or inaccuracies of the force-field description in the MD potential.

To investigate the impact of dispersed fission gas on the thermal conductivity, we employed similar MD simulations with increasing concentrations of Xe from $0.33 \%$ to $1.0 \%$. According to the our fission gas behavior model, the dispersed Xe concentration in the fuel during normal operating conditions would typically reach a value of $1.0 \%$ in the outer region of the fuel (below $1200 \mathrm{~K}$ ) at a burn-up of approximately $40 \mathrm{MWD} / \mathrm{kgU}$. At the higher temperatures present in the central region of the fuel, the increased importance of intra-granular diffusional release and trapping shifts the attainment of $1.0 \%$ dispersed Xe concentrations to higher burn-ups (e.g., about $85 \mathrm{MWd} / \mathrm{kgU}$ at $1400 \mathrm{~K}$ ). As with the single crystal thermal conductivity, we cor- rected these results using the spin scattering term from the Callaway model. The MD results clearly show that Xe has a large impact, as shown in Fig. 2, with a large difference between the thermal conductivity of pure $\mathrm{UO}_{2}$ and that with $c_{X e}=0.0033$. The increased reduction of the thermal conductivity for $c_{X e}=0.0067$ and $c_{X e}=0.01$ is smaller, though still significant.

If we employ the value for $K_{X e}$ calculated with Eq. (18) in Eq. (16), the predicted thermal conductivity compares fairly well with the MD data for concentrations $c_{X e}=0.0067$ and $c_{X e}=0.01$, but it predicts thermal conductivities that are too high for $c_{X e}=0.0033$, as shown in Fig. 2(a). The reason for this inaccuracy of the model at low concentrations is unclear and needs to be investigated in future work. As an alternative to the analytical model, we modify Eq. (16) to be

$$
k_{L}(T)=\left(A+B T+C T^{2}+K_{X e} c_{X e}^{d}\right)^{-1} .
$$

and fit the values of the coefficient $K_{X e}$ and the exponent $d$ to the MD data. The fitted values were $K_{X e}=1.5 \mathrm{mK} / \mathrm{W}$ and $d=0.45$ and resulted in better predictions than the coefficient from Eq (18), as shown in Fig. 2(b). Note that Eq. (19) is simply a fit to the MD data and does not have a theoretical justification.

We do not use the values for the $A, B$, and $C$ coefficients fitted to the MD simulation results in the final thermal conductivity model. Rather, we use the coefficients fitted to experimental data from Fink [1] with the dispersed Xe scattering term in Eq. (19). The values of the thermal conductivity based on the fit from Fink [1] with our additional phonon scattering term for dispersed Xe concentration is shown in Fig. 2(c).

\subsection{Impact of Intragranular Bubbles}

The impact of porosity on thermal conductivity is often accounted for using analytical mixing models such as the Maxwell-Eucken equation, which takes the form of a multiplier to the thermal conductivity of the form

$$
\kappa_{M E}=\frac{1-p}{1+0.5 p},
$$

where $p$ is the porosity. However, mixing models only consider changes in the local thermal conductivity, and are valid in the regime of the phonon mean free path $l$ is much greater than the pore radius $r$. In the regime where these quantities are 


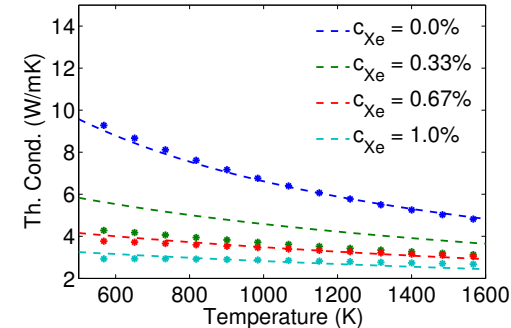

(a)

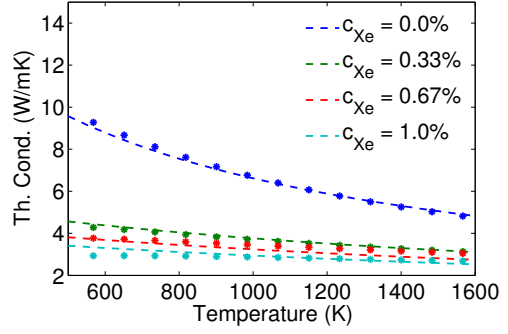

(b)

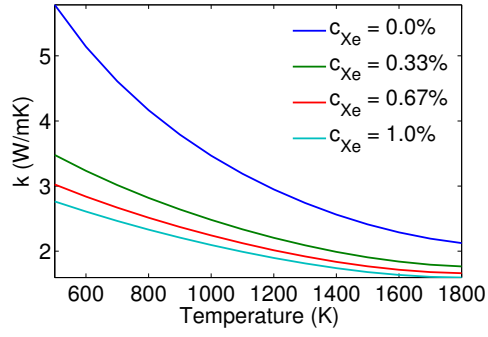

(c)

Figure 2: Impact of dispersed Xe on the $\mathrm{UO}_{2}$ thermal conductivity for various gas concentrations, where the dots show the corrected MD results and the dashed line shows the fit to the data. (a) shows the model predictions when $K_{X e}$ was calculated using Eq. (18) and (b) shows the prediction when the coefficient and exponent are fit to the MD results. The impact of dispersed Xe on the thermal conductivity from Fink [1] according to the model is shown in (c).

compatible, or even $l>r$, more elaborate models are required. The intragranular fission gas bubbles within the fuel grains remain small, due to radiation induced resolution of gas atoms, typically ranging from a radius of $0.5 \mathrm{~nm}$ to $2 \mathrm{~nm}$, and thus fall into this regime. In Nichenko and Staicu [30] and Lee et al. [31], MD simulations were used to quantify the impact of small bubbles on the thermal conductivity. Both found that mixing models, such as the Maxwell-Eucken equation, under predict the impact of these small bubbles on the fuel thermal conductivity. Lee et al. [31] also compared the MD calculated thermal conductivity to that predicted by a recent model from Alvarez et al. [32] that considers the impact of phonon scattering, i.e.

$$
\kappa_{A}=\frac{1}{\frac{1}{k_{M E}}+\frac{9 l^{2}}{2 r^{2}} \frac{p(1+3 \sqrt{p / 2})}{1+\left(0.864+0.29 e^{-1.25 \frac{r}{l}}\right) \frac{l}{r}}}
$$

They found that the Alvarez model was the best comparison to the MD data. However, it is clear that the phonon scattering is only significant for smaller bubbles. Thus, there is a critical radius above which the Maxwell-Eucken accurately captures the impact of the pores on the thermal conductivity. To determine the critical radius, we compare the multiplier from Eq. (20) to that from Eq. (21) as a function of bubble radius for a given porosity, as shown in Fig. 3(a). We then computed the threshold radius as a function of porosity for which the values predicted by the two models varied by $5 \%, 1 \%$, and $0.1 \%$ (Fig. $3(\mathrm{~b})$ ). Thus, for a porosity of 0.02 , pores with a radius of $5 \mathrm{~nm}$ will have an impact on the thermal conductivity within $5 \%$ of the value predicted by the Maxwell-Eucken equation, with a radius of $10 \mathrm{~nm}$ will be within $1 \%$, and with a radius of $25 \mathrm{~nm}$ will be within $0.1 \%$. Because intragranular porosity typically has a radius of less then $2 \mathrm{~nm}$, Eq. (21) (the Alvarez model) should be used to predict its impact.

\subsection{Modeling Impact of GB Bubbles on Thermal Conductivity}

GBs restrict heat flow due to their increased lattice disorder. The effect is quantified with a GB thermal resistance or Kapitza resistance $R_{k}$ [33]. When a GB is laden with fission gas bubbles, this further increases the thermal resistance of the GB, which can be quantified with an effective GB thermal resistance $R_{k}^{\prime}\left(f_{c}\right)$. The effect of the bubbleladen GB on thermal conductivity is quantified using a multiplier to the thermal conductivity, i.e.

$$
\kappa_{G B}=\frac{1}{1+k_{0} R_{k}^{\prime}\left(f_{c}\right) / d},
$$

where $d$ is the grain size and $k_{0}$ is the unirradiated thermal conductivity of $\mathrm{UO}_{2}$. Several efforts have been made to determine the effective GB thermal resistance as a function of the fission gas fractional coverage using fits to mesoscale heat conduction simulations of various $2 \mathrm{D}$ grain structures $[6,34,35]$. However, it is well known that 2D simulations over predict the impact of secondary phases on the effective thermal conductivity [36], thus 3D simulations have also been applied to quantify the effective GB thermal resistance [37]. However, in past work models describing the impact of the GB fractional coverage on GB thermal resistance have been empirical fits to the simulation results $[34,35,37]$, or semi-empirical models [6]. In this work, we develop an analytical constitutive model of the effective GB thermal resistance, and 


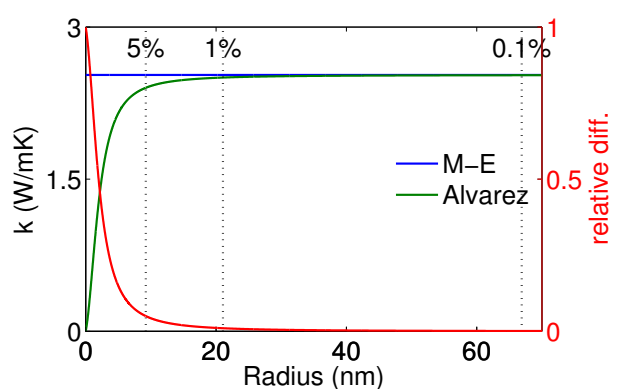

(a)

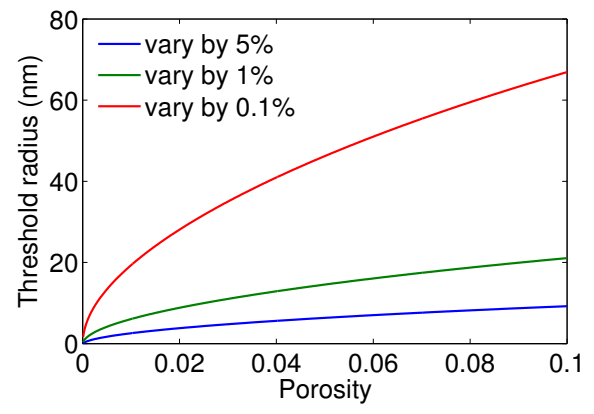

(b)

Figure 3: Determination of the threshold radius at which phonon scattering significantly impacts the effect of pores on thermal conductivity, where (a) shows the comparison of the Maxwell-Eucken equation to the Alvarez equation and (b) plots the threshold bubble radius for which the two models vary by less then the specified percent.

compare to the 3D bicrystal results from Millett et al. [37] to parameterize the model.

To develop an analytical model of the thermal resistance of a bubble-laden GB, we approximate the real geometry with thermal resistors and then equate the approximate system to the GB thermal resistance equation. For example, if we approximate a GB as a block of material with crosssectional area $A$, thickness $l_{G B}$, and thermal conductivity $k_{G B}$ embedded in a bulk material with grain size $d$, we can approximate it as two thermal resistors in series, where the effective thermal resistance $R_{e f f}=R_{0}+R_{G B}$. The thermal resistance in the bulk $R_{0}=d / A k_{0}$ and in the GB $R_{G B}=l_{G B} / A\left(1 / k_{G B}-1 / k_{0}\right)$, where we remove the conductivity of the bulk material across the GB and add in that of the GB. If we combine these ex-

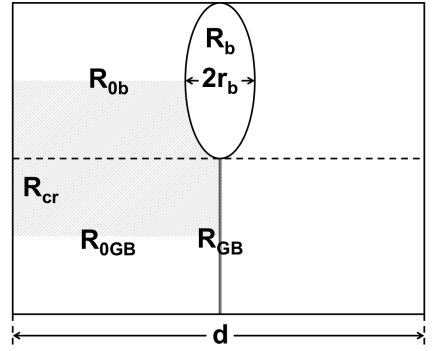

(a)

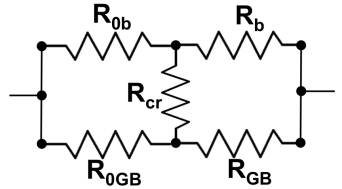

(b)
Figure 4: Simplified system used to approximate the effective GB thermal resistance as a function of the GB coverage, where the microstructure is represented with one bubble and one GB section, as shown in (a). The effective thermal resistance is computed using the five thermal resistors shown in (b).

pressions, we obtain

$$
\begin{aligned}
\frac{d}{A k_{e f f}} & =\frac{d}{A k_{0}}+\frac{l_{G B}}{A}\left(\frac{1}{k_{G B}}-\frac{1}{k_{0}}\right) \\
\frac{1}{k_{e f f}} & =\frac{1}{k_{0}}+\frac{l_{G B}}{d}\left(\frac{1}{k_{G B}}-\frac{1}{k_{0}}\right)
\end{aligned}
$$

The effective GB thermal resistance is obtained by equating this expression to that for the Kaptiza resistance model and solving for the Kapitza resistance, i.e.

$$
\begin{aligned}
\frac{1}{k_{0}}+\frac{R_{k}}{d} & =\frac{1}{k_{0}}+\frac{l_{G B}}{d}\left(\frac{1}{k_{G B}}-\frac{1}{k_{0}}\right) \\
R_{k} & =l_{G B}\left(\frac{1}{k_{G B}}-\frac{1}{k_{0}}\right) .
\end{aligned}
$$

To develop a model for GBs laden with bubbles, we take a similar approach to that used in the previous simple example of a single GB. However, now we consider a system with one bubble and a single flat GB, as shown in Fig. 4(a). We represent it with a series of five resistors, as shown in Fig. 4(b), where the resistor used to represent each area is labeled in Fig. 4(a). The bubble is treated as resistor $R_{b}$ and the $\mathrm{UO}_{2}$ on either side of the bubble is resistor $R_{0 b}$, such that

$$
\begin{aligned}
R_{b} & =\frac{2 r_{b}}{f_{c} A}\left(\frac{1}{k_{b}}-\frac{1}{k_{0}}\right) \\
R_{0 b} & =\frac{d}{f_{c} A k_{0}},
\end{aligned}
$$

where the bubble is treated as a block of width $2 r_{b}$, $r_{b}$ is the bubble radius, and the bubble cross sectional area is defined in terms of the total cross sectional area $A$ and the fractional coverage, i.e. $f_{c} A$. 
The thermal conductivity of the bubble is equal to the conductivity of Xe at $300 \mathrm{~K}, k_{b}=5.65 \mathrm{~mW} / \mathrm{mK}$ [38]. The GB is resistor $R_{G B}$ and the $\mathrm{UO}_{2}$ on either side of the GB is resistor $R_{0 G B}$, such that

$$
\begin{aligned}
R_{G B} & =\frac{R_{k}}{\left(1-f_{c}\right) A} \\
R_{0 G B} & =\frac{d}{\left(1-f_{c}\right) A k_{0}},
\end{aligned}
$$

where the resistance of the GB is defined by the Kapitza resistance $R_{k}$ over the portion of the area not covered by fission gas $\left(\left(1-f_{c}\right) A\right)$. We account for the portion of the heat that can cross between the $\mathrm{UO}_{2}$ on either side of the bubble and on either side of the GB by adding an additional resistor $R_{c r}$. The effective thermal resistance across the system of resistors was determined by applying Kirchoff's laws to obtain

$$
\begin{aligned}
R_{e f f}= & \frac{R_{e f f}^{1}}{R_{e f f}^{2}} \\
R_{e f f}^{1}= & R_{0 b} R_{c r} R_{0 G B}+R_{0 b} R_{c r} R_{G B}+ \\
& R_{0 b} R_{0 G B} R_{b}+R_{0 b} R_{0 G B} R_{G B}+ \\
& R_{0 b} R_{b} R_{G B}+R_{c r} R_{0 G B} R_{b}+ \\
& R_{c r} R_{b} R_{G B}+R_{0 G B} R_{b} R_{G B} \\
R_{e f f}^{2}= & R_{0 b} R_{c r}+R_{0 b} R_{b}+R_{0 b} R_{G B}+ \\
& R_{c r} R_{0 G B}+R_{c r} R_{b}+R_{c r} R_{G B}+ \\
& R_{0 G B} R_{b}+R_{0 G B} R_{G B} .
\end{aligned}
$$

The effective Kapitza resistance is obtained in the manner shown in Eq. (24),

$$
R_{k}^{\prime}=A R_{e f f}-\frac{d}{k_{0}} .
$$

The value of the resistor $R_{c r}$ allows for some three dimensional (3D) heat transfer in what would otherwise be a one dimensional heat transfer model. When $R_{c r}=0$ heat can transfer freely between the bubble and GB regions of the domain resulting in a lower bound on the effective thermal conductivity of the system. When $R_{c r}$ is large, any heat that passes through $R_{G B 0}$ must also pass through $R_{G B}$ and heat that passes through $R_{b 0}$ must pass through $R_{b}$. This results in an upper bound on the effective thermal conductivity. In reality, the value of $R_{c r}$ is impacted by the bulk conductivity and the fractional coverage, but is difficult to define analytically. However, a value of $R_{c r}$ can be fit by comparing to $3 \mathrm{D}$ heat conduction simulation results. Here, we fit $R_{c r}$ by comparing to the

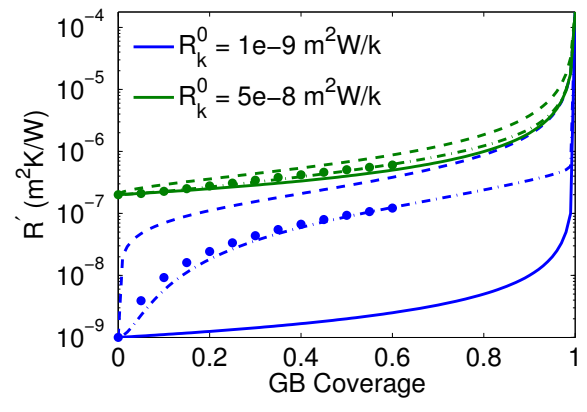

Figure 5: Comparison of the effective Kapitza resistance determined from the analytical model to that from the 3D simulations conducted in Millett et al. [37]. The 3D simulation results are shown as points, the lower bound as a solid line, the upper bound as a dashed line, and the model using the fit value $R_{c r}=5.0 \times 10^{-7} \mathrm{~m}^{2} \mathrm{~K} / \mathrm{W}$ as a dash-dot line.

results from Millett et al. [37] in which a lenticular bubble was placed on a flat GB and the effective thermal conductivity was determined using a heat conduction simulation solved with the finite element method in the mesoscale fuels performance code MARMOT [5]. The effective Kapitza resistance was obtained from these calculations for various GB Kapitza resistances, GB fractional coverages, and bubble radii. The comparison between the analytical model and the 3D simulation results are shown in Fig. 5. The upper and lower bounds (defined when $R_{c r}$ is large or $R_{c r}=0$, respectively) bracket the simulation predictions as expected. A value of $R_{c r}=5.0 \times 10^{-7} \mathrm{~m}^{2} \mathrm{~K} / \mathrm{W}$ provides the best fit with the simulation results. Though the results for only two values of $R_{k}$ are shown in Fig. 5, similar comparisons were obtained for all values used in Millett et al. [37].

\section{Implementation in the BISON Fuel Per- formance Tool}

The fission gas analytical thermal conductivity model accounts for the impact of dispersed fission gas (in terms of the concentration $c_{f g}$ ), intragranular bubbles (in terms of the porosity $p_{i g}$ ), and GB bubbles (in terms of the GB fractional coverage $f_{c}$ ) according to the equation

$$
k=\kappa_{A}\left(p_{i g}\right) \kappa_{G B}\left(f_{c}\right)\left(k_{L}\left(t, c_{X e}\right)+k_{A P}(t)\right)
$$

where $t=T(K) / 1000, \kappa_{A}$ is defined in Eq. (21), $\kappa_{G B}$ is defined in Eq. (22), $k_{L}$ is defined in Eq. (19), and $k_{A P}$ is defined in Eq. (13). This model directly couples to the fission gas release model [7], where 
$c_{f g}$ is calculated according to Eq. (11), $p_{i g}$ according to Eq. (9), and $f_{c}$ according to Eq. (10). Thus, phonon scattering on dispersed gas is accounted for directly in the thermal conductivity equation and the contributions of gas bubbles (both intra- and intergranular) are included using multipliers to the thermal conductivity equation.

We conduct two studies of the impact of this thermal conductivity model on macroscale fuel performance. First, we recorded the conditions from a fuel rodlet simulation, and used them with the model to investigate the relative importance of the different distributions of fission gas. We then use the model in the BISON tool to simulate the fuel performance in four reactor experiments.

\subsection{Investigation of the Relative Importance of the Gas Distributions}

To determine the relative importance of the distributions of fission gas on the overall reduction in thermal conductivity, we simulate the behavior of a ten pellet fuel rodlet experiencing typical pressurized water reactor conditions for 2.5 years using the BISON tool. The fuel rodlet is represented in $2 \mathrm{D}$ assuming axisymmetry and the ten pellets are smeared together to simplify the simulation. The simulation uses the full set of empirical models defining all of the material behavior in the fuel and cladding [9].

The temperature, grain radius, and the microstructure variables defining the fission gas behavior were recorded throughout the simulation at the axial mid-plane, both at the pellet center and the outer radius. The fission gas thermal conductivity model was used to calculate the impact of the different distributions of fission gas on the thermal conductivity, as well as the combined effect, as shown in Fig. 6. For the intergranular bubble contribution, we employed the fit value for $R_{c r}$. On the fuel edge, the dispersed fission gas accounts for an average of $70.5 \%$ of the reduction in the thermal conductivity due to fission gas, with intragranular bubbles accounting for $28.0 \%$ and GB bubbles accounting for $1.5 \%$ since the temperature is too low for a significant number of GB bubbles to form. In the center of the fuel, the higher temperature results in lower concentration of dispersed gas and the formation of large GB bubbles. The overall reduction in thermal conductivity due to fission gas was $25 \%$ less in the fuel center than on the outer edge, probably due to fission gas release. Dispersed gas atoms account for an average of $62.7 \%$ of the thermal conductivity reduction, intragranular bubbles account for $30.0 \%$, and GB bubbles account for $7.3 \%$. Thus, the dispersed fission gas has the largest impact on the thermal conductivity in both fuel locations.

To quantify the sensitivity of the GB bubble model to the value for $R_{c r}$, we calculated the impact of GB bubbles at the fuel center using the lower bound $\left(R_{c r}=0\right)$, the fit value $\left(R_{c r}=5 \times 10^{-7}\right.$ $\left.\mathrm{m}^{2} \mathrm{~K} / \mathrm{W}\right)$ and the upper bound $\left(R_{c r}>1 \mathrm{~m}^{2} \mathrm{~K} / \mathrm{W}\right)$, shown in Fig. 6(c). The lower bound predicts no significant impact due to intergranular bubbles while the upper bound predicts a very significant impact, larger than all other contributions. Thus, the model is sensitive to $R_{c r}$ and its value should be maintained on the order of the fit value.

\subsection{Simulation of Reactor Tests}

The fission gas thermal conductivity model was implemented in BISON and coupled to the fission gas release model. To evaluate the accuracy of the model, we have used it to model the fuel behavior in four reactor test experiments and compared to experimental data and simulation results using empirical models. Our model does not fully define the decrease in thermal conductivity during reactor operation, as other effects of radiation damage were not considered, such as the generation of point defects, stoichiometry change, and the impact of precipitated and dissolved fission products [2]. Thus, we expect the model to over predict the thermal conductivity and predict lower temperatures. However, future work will determine the impact of these other contributions to complete the microstructurebased thermal conductivity model.

The Ris $\varnothing$ AN3 experiment conducted at the Ris $\varnothing$ DR3 water-cooled HP1 rig utilized a refabricated rod from the Biblis A pressurized water reactor (PWR) [39, 40]. The mother rod, CB8, was irradiated over four reactor cycles up to about 41 $\mathrm{MWd} / \mathrm{kgU}$, and re-fabricated to a shorter length with Helium as the fill gas. The re-fabricated rod, CB8-2R, was instrumented with a fuel centerline thermocouple and a pressure transducer and ran through a series of transient power conditions (see [39] for the power history). The fuel centerline temperature and fission gas release were used for comparison. The thermocouple temperature predicted using the fission gas thermal conductivity model is lower than the measured value, as expected. The predictions were very similar to those using the 


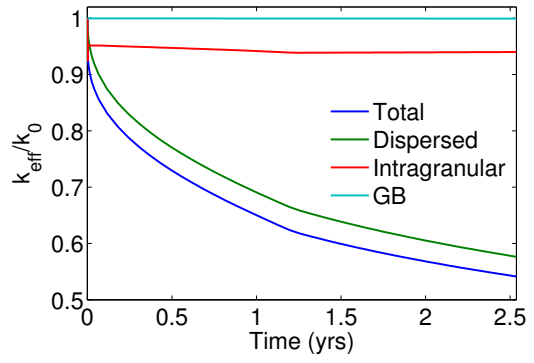

(a)

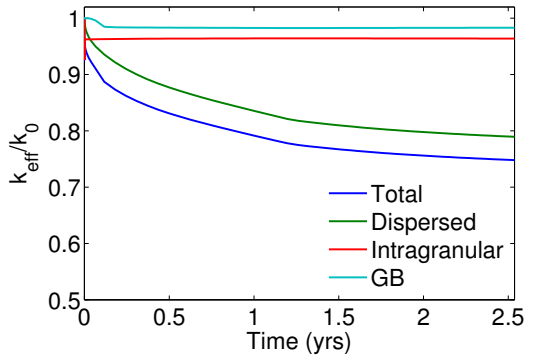

(b)

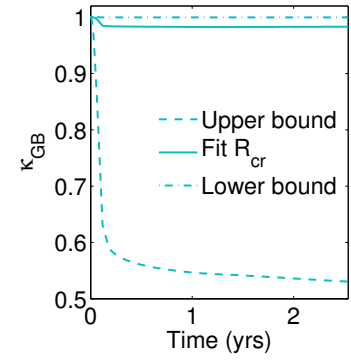

(c)

Figure 6: The impact of fission gas on the thermal conductivity as predicted by our model, using conditions outputted from a ten pellet rodlet simulation, where the behavior at the fuel outer edge is shown in (a) and in the fuel center in (b). The dispersed fission gas has the largest impact on the thermal conductivity both at the fuel edge and in the center. A comparison of the impact of GB bubbles at the fuel center for different values of $R_{c r}$ is shown in (c).

Electric Power Research Institute Nuclear Fuel Industry Research (NFIR) empirical model [4] (see Fig. 7(a)). The fission gas release predictions were also below the measured values but consistent with those using the NFIR model (Fig. 7(e)).

The Ris $\varnothing$ AN4 experiment also utilized a refabricated rod at the Ris $\varnothing$ DR3 rig [39, 41]. The mother rod, CB7, was irradiated over four reactor cycles then re-fabricated to a shorter length with $\mathrm{Xe}$ as the fill gas. The re-fabricated rod, CB7-2R, was fitted with a fuel centerline thermocouple and a pressure transducer and ran through a series of transients [39]. Again, the thermocouple temperature and fission gas release were used for comparison. The predicted temperature was lower than the data and was very close to the predictions using the NFIR model (Fig. 7(b)). The fission gas predictions were not as close to the data as the AN3 assessment (Fig. 7(f)).

The Risø II3 experiment conducted at the Ris $\emptyset$ DR3 rig utilized a re-fabricated rod from the Millstone-1 BWR [42, 43]. The mother rod, STR014, was irradiated over three reactor cycles up to about $14.5 \mathrm{MWd} / \mathrm{kgU}$, and re-fabricated to a shorter length. The re-fabricated rod, II3 (STR014-3R), was instrumented with a fuel centerline thermocouple and a pressure transducer and ran through a transient power history [42]. The thermocouple temperature and fission gas release were used for comparison. For this case, the predicted thermocouple temperatures using the fission gas thermal conductivity model were significantly higher than the those from the NFIR model and were close to the experimental data, as shown in Fig. 7(c). The fission gas release predictions were significantly higher than the NIFR predictions and the values calculated from the measured plenum pressure (Fig. $7(\mathrm{~g})$ ). Note that while the results for this experiment appear to be significantly more accurate than for the Ris $\varnothing-\mathrm{AN} 3$ and AN4 experiments, this may be misleading. Once the thermal conductivity model is complete and the contributions of additional defects are included, the temperature is likely to be over-predicted. The difference between the II 3 and the AN3 and AN4 results could be due a number of possibilities, including error in the experimental measurements, inaccuracies in the characterization of the initial irradiation, or missing mechanisms in the fission gas release model.

The US PWR $16 \times 16$ lead test assembly extended burn-up demonstration was conducted during the 1980's in a US commercial pressurized water reactor (PWR) [42]. The purpose of this series of experiments was to increase final discharge burn-up and to demonstrate improved fuel utilization through more efficient fuel management. TSQ002 was a full length fuel rod with standard (solid) fuel pellets that was discharged at a burnup of approximately $58 \mathrm{MWd} / \mathrm{kgU}$. The rod was not instrumented, therefore we compare the temperature predictions to the predictions from the TRANSURANUS [44, 45] and FALCON [46] codes. For fission gas release, we compare to the final gas release measured by puncturing the rod after irradiation. Fuel centerline temperature calculated by the fission gas thermal conductivity model predicted higher temperatures than the NFIR model initially, but after a burn-up of $20 \mathrm{MWd} / \mathrm{kgU}$ the temperature predicted by the NFIR model increased above the fission gas model. The BISON 


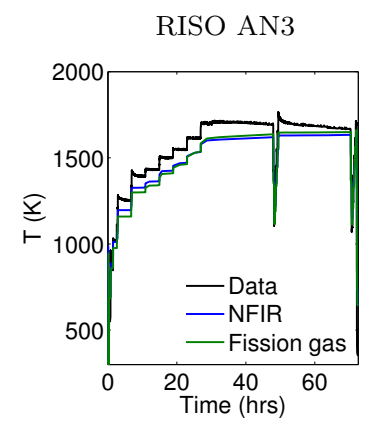

(a)

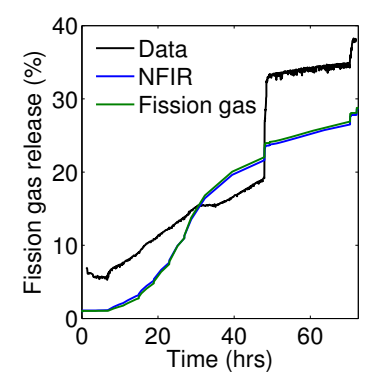

(e)

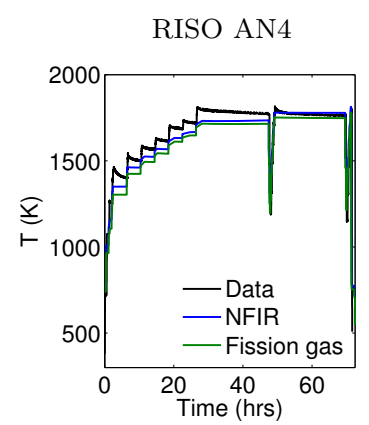

(b)

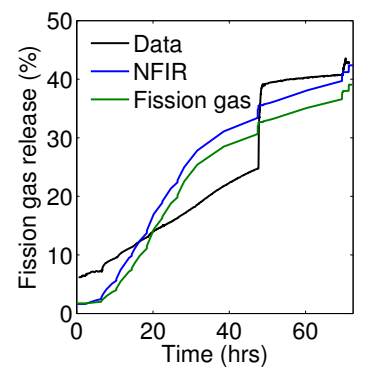

(f)
RISO II3

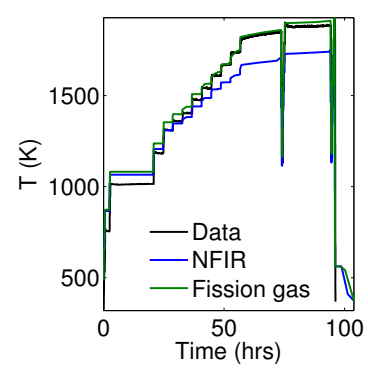

(c)

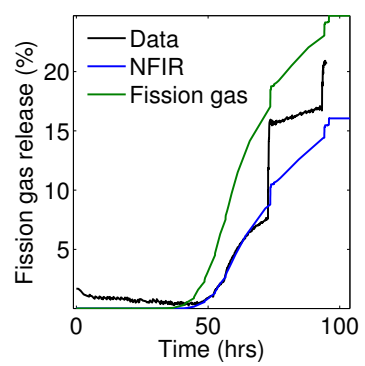

(g)
US PWR TSQ002

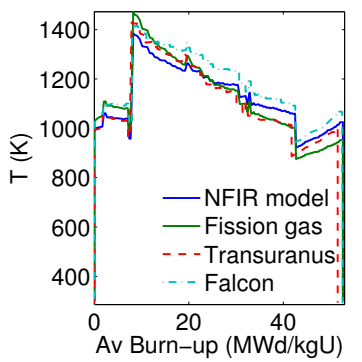

(d)

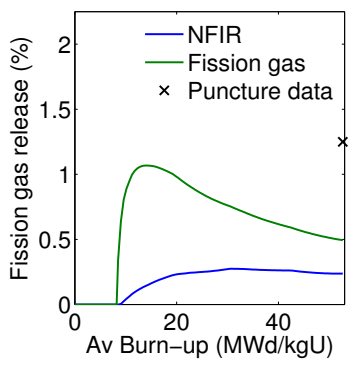

(h)

Figure 7: Predicted centerline temperature and fission gas release for four assessment cases, where the temperature predictions are shown at the top and the fission gas release at the bottom. The results for the Ris $\varnothing$ AN3 test case are shown in (a) and (e), for the Ris $\varnothing$ AN4 test case are shown in (b) and (f), for the Ris $\varnothing$ II-3 test are shown in (c) and (g), and for the US PWR TSQ-002 test in (d) and (h). Note that the only data available for the US PWR TSQ-002 test is the final fission gas release collected from puncturing the cladding after irradiation.

predictions using the fission gas thermal conductivity model were close to the TRANSURANUS predictions, while the FALCON predictions were the highest [42]. The fission gas release predicted by the model were closer to the measured fission gas release than that from the NFIR model.

Overall, the new model predicted the fuel temperatures with reasonable accuracy when comparing to data and to fuel performance codes using empirical models. This was achieved without any fitting to fuel centerline thermocouple data, but just taking information from lower length-scale simulations, as discussed in this work. However, as the model currently only includes fission gas contributions, when the contributions from other fission products and irradiation damage are added, the predicted temperature will increase. This increase will increase the temperature predictions for the Ris $\varnothing$-II3 above the measured temperature. All calculations in this paper treated only Xe atoms for the fission gas, which could lead to an over prediction of the impact of the fission gas. In addition, because the dispersed gas atoms have the largest impact on the thermal conductivity (Fig. 6), the model predictions are sensitive to the accuracy of the dispersed gas atom concentration from the fission gas release model.

\section{Conclusions}

Accurately predicting the impact of irradiation damage on fuel thermal conductivity is essential to accurately predicting fuel performance. In this work, we have developed a model that accounts for the impact of fission gas on the fuel thermal conductivity. This thermal conductivity model directly couples to the fission gas release model. To quantify the impact of fission gas on the fuel thermal conductivity, we have used atomistic and mesoscale modeling approaches to inform the development of an analytical model. The impact of dispersed fission gas atoms within the $\mathrm{UO}_{2}$ matrix was quantified using MD simulation results that were corrected to account for the large phonon-spin scattering effects. We found that the intragranular fission gas bubbles are small enough to require that phonon scatter- 
ing effects be included, necessitating the use of the model from Alvarez et al. [32]. To determine the impact of GB fission gas bubbles, we developed a model using five thermal resistors by comparing to 3D mesoscale heat conduction simulation results. A detailed comparison of these three contributions showed that the dispersed fission gas atoms have the largest impact on the fuel thermal conductivity, accounting for an average of $70.5 \%$ of the total thermal conductivity reduction at the fuel edge and $62.7 \%$ at the fuel center. We implemented the fission gas thermal conductivity model in the BISON fuel performance code and coupled it to the fission gas release model. To evaluate the model, we compared to four reactor experiments. The model does not account for other fission products or radiation damage, but the predictions were reasonable without having been fit to fuel thermocouple data. Future work will add the contributions of other fission products and radiation damage to complete a full thermal conductivity model.

\section{Acknowledgements}

This work was funded by the Department of Energy Nuclear Energy Advanced Modeling and Simulation program. This manuscript has been authored by Battelle Energy Alliance, LLC under Contract No. DE-AC07-05ID14517 with the US Department of Energy. The United States Government retains and the publisher, by accepting the article for publication, acknowledges that the United States Government retains a nonexclusive, paid-up, irrevocable, world-wide license to publish or reproduce the published form of this manuscript, or allow others to do so, for United States Government purposes. Los Alamos National Laboratory, an affirmative action/equal opportunity employer, is operated by Los Alamos National Security, LLC, for the National Nuclear Security Administration of the U.S. Department of Energy under Contract No. DEAC52-06NA25396.

\section{References}

[1] J. K. Fink, J. Nucl Materials 279 (2000) 1-18.

[2] P. Lucuta, H. Matzke, I. Hastings 232 (1996) 166-180.

[3] C. Ronchi, M. Sheindlin, D. Staicu, M. Kinoshita, Journal of nuclear materials 327 (2004) 58-76.

[4] A. Marion (NEI) letter dated June 13, 2006 to $H$. N. Berkow (USNRC/NRR), Safety Evaluation by the Office of Nuclear Reactor Regulation of Electric Power Research Institute (EPRI) Topical Report TR-1002865,
Topical Report on Reactivity Initiated Accidents: Bases for RIA Fuel rod Failures and Core Coolability Criteria, http://pbadupws.nrc.gov/docs/ML0616/ML061650107.pdf, 2006.

[5] M. Tonks, D. Gaston, P. Millett, D. Andrs, P. Talbot, Comp. Mat. Sci. 51 (2012) 20-29.

[6] M. R. Tonks, Y. Zhang, S. Biner, P. C. Millett, X. Bai, Acta Materialia 61 (2013) 1373-1382.

[7] G. Pastore, L. Luzzi, V. Di Marcello, P. Van Uffelen, Nuclear Engineering and Design 256 (2013) 75-86.

[8] G. Pastore, L. P. Swiler, J. D. Hales, D. M. Perez, B. W. Spencer, L. Luzzi, P. V. Uffelen, R. L. Williamson, Journal of Nuclear Materials 456 (2015) 398-408.

[9] R. L. Williamson, J. D. Hales, S. R. Novascone, M. R. Tonks, D. R. Gaston, C. J. Permann, D. Andrs, R. C. Martineau, Journal of Nuclear Materials 423 (2012) 149-163.

[10] M. Speight, Nuclear Science and Engineering 37 (1969) 180-185.

[11] R. White, M. Tucker 118 (1983) 1-38.

[12] G. Pastore, D. Pizzocri, J. D. Hales, S. R. Novascone, D. M. Perez, B. W. Spencer, R. L. Williamson, P. Van Uffelen, L. Luzzi, in: Proc. of the Enlarged Halden Programme Group Meeting, Roros, Norway, September $7-12$.

[13] C. Ronchi, M. Sheindlin, M. Musella, G. Hyland, Journal of Applied Physics 85 (1999) 776-789.

[14] D. Ecsedy, P. Klemens, Physical Review B 15 (1977) 5957.

[15] A. Eucken, Forsch, Gebiete Ingenieur 4 (1932) 6.

[16] I. Fritz, Journal of Applied Physics 47 (1976) 43534358.

[17] P. Jund, R. Jullien, Arxiv preprint cond-mat/9903033 (1999).

[18] F. Müller-Plathe, The Journal of chemical physics 106 (1997) 6082-6085.

[19] T. Ikeshoji, B. Hafskjold, Molecular Physics 81 (1994) 251-261.

[20] S. J. Plimpton, J. Comp. Phys. 117 (1995) 1-19.

[21] X. Zhou, S. Aubry, R. Jones, A. Greenstein, P. Schelling, Physical Review B 79 (2009) 115201.

[22] P. K. Schelling, S. R. Phillpot, P. Keblinski, Physical Review B 65 (2002) 144306.

[23] K. Gofryk, S. Du, C. Stanek, J. Lashley, X.-Y. Liu, R. Schulze, J. Smith, D. Safarik, D. Byler, K. McClellan, et al., Nature communications 5 (2014).

[24] G. Busker, A. Chroneos, R. W. Grimes, I.-W. Chen, Journal of the American Ceramic Society 82 (1999) 1553-1559.

[25] T. Watanabe, S. B. Sinnott, J. S. Tulenko, R. W. Grimes, P. K. Schelling, S. R. Phillpot, Journal of Nuclear Materials 375 (2008) 388-396.

[26] T. Uchida, T. SUNAOSHI, M. KATO, K. Konashi, Progress in Nuclear Science and Technology 2 (2010) 598-602.

[27] J. Moore, D. McElroy, Journal of the American Ceramic Society 54 (1971) 40-46.

[28] J. Callaway, Physical Review 113 (1959) 1046.

[29] N. G. Verma, Physical Review B 6 (1972) 3509.

[30] S. Nichenko, D. Staicu, Journal of Nuclear Materials 454 (2014) $315-322$.

[31] C.-W. Lee, A. Chernatynskiy, P. Shukla, R. Stoller, S. Sinnott, S. Phillpot, Journal of Nuclear Materials 456 (2015) $253-259$.

[32] F. Alvarez, D. Jou, A. Sellitto, Applied Physics Letters 
97 (2010) 033103.

[33] P. Kapitza, J. Phys. (Moscow) 4 (1941) 181.

[34] P. C. Millett, M. Tonks, J. Nucl. Mater. 412 (2011) 281 -286 .

[35] K. Chockalingam, P. Millett, M. Tonks, Journal of Nuclear Materials 430 (2012) 166-170.

[36] K. Bakker, H. Kwast, E. Cordfunke, Journal of Nuclear Materials 226 (1995) 128-143.

[37] P. C. Millett, M. R. Tonks, K. Chockalingam, Y. Zhang, S. Biner, Journal of Nuclear Materials 439 (2013) 117122 .

[38] E. Thornton, Proceedings of the Physical Society 76 (1960) 104

[39] IAEA, Fuel Modelling at Extened Burnup (FUMEXII): Report of a Coordinated Research Project 20022007, Technical Report IAEA-TECDOC-1687, International Atomic Energy Agency, 2002-2007.

[40] The Third Ris $\varnothing$ Fission Gas Project: Bump Test AN3 (CB8-2R), Technical Report Ris $\varnothing-F G P 3-A N 3$, Ris $\varnothing$, 1990.

[41] The Third Ris $\varnothing$ Fission Gas Project: Bump Test AN4 (CB7-2R), Technical Report Ris $\varnothing-F G P 3-A N 4$, Ris $\varnothing$, 1990.

[42] IAEA, Improvement of Computer Codes Used for Fuel Behaviour Simulation (FUMEX-III): Report of a Coordinated Research Project 2008-2012, Technical Report IAEA-TECDOC-1697, International Atomic Energy Agency, 2008-2012.

[43] The Third Ris $\varnothing$ Fission Gas Project: Bump Test II3 (STR014-3R), Technical Report Ris $\varnothing-F G P 3-I I 3$, Ris $\varnothing$, 1990.

[44] K. Lassmann, Journal of Nuclear Materials 188 (1992) 295-302.

[45] K. Lassmann, H. Blank, Nuclear Engineering and Design 106 (1988) 291-313.

[46] Y. Rashid, R. Dunham, R. Montgomery, Fuel Analysis and Licensing Code: FALCON MOD01, Technical Report EPRI 1011308, Electric Power Research Institute, 2004. 